\title{
... Und führe uns nicht in Versuchung, sondern erlöse uns von dem Bösen ... Gedanken zu Eginald Schlattners Text Ja nicht ja. Walther Gottfried Seidner zum 80. ${ }^{1}$
}

\author{
Andreea DUMITRU \\ Dr., Sibiu/Hermannstadt. \\ E-Mail: andreeadumitruiacob@gmail.com
}

\begin{abstract}
The story "Ja nicht ja" was written specifically for the volume "Der siebenbürgische Voltaire. Walther Gottfried Seidner zum 80 . Geburtstag" by the famous novelist Eginald Schlattner. It brings the communist regime and the Department of State Security into the focus of the reader. During a meeting in the early 1990s attended by evangelical Lutheran priests of Augustan Confession a young priest admitted that he was a collaborator of the State Security, and thus managed to take over the burden of being an informant on the shoulders of others. Father Walther Gottfried Seidner, who was also threatened, managed to avoid State Security at any price, and understanding the situation of the young priest takes his defense.
\end{abstract}

Key words: Walther Seidner, Eginald Schlattner, comunisms, State Security, priest

Die Erzählung Ja nicht ja. Walther Gottfried Seidner zum 80. von Eginald Schlattner ist am 4. Mai 2018 (Teil I) und am 08. Mai 2018 (Teil II) in der Allgemeinen Deutschen Zeitung für Rumänien erschienen. Derselbe Text erschien auch auch in dem Band von Andreea Dumitru; Sunhild Galter (Hgg.): Der siebenbürgische Voltaire. Walther Gottfried Seidner zum 80 Geburtstag. Sibiu/Hermannstadt: Honterus Verlag 2018, ISBN 978-606-8573-75-5. 


\section{Einleitung:}

Der vorliegende Text wurde von dem bekannten Pfarrer und Schriftsteller Eginald Schlattner speziell für die Festschrift Der siebenbürgische Voltaire. Walther Gottfried Seidner zum 80. Geburtstag geschrieben; es handelt sich um einen literarischen Bericht, der das Thema der jüngsten Vergangenheit in den Mittelpunkt unserer Aufmerksamkeit rückt. Der Einfluss des rumänischen Sicherheitsdienstes in den Zeiten der Kommunistischen Diktatur wird hier aus der Perspektive der evangelischen Pfarrer aufgezeigt. Dieser Text hat eine besondere Bedeutung, weil er die siebenbürgischen Persönlichkeiten Schlattner und Walther G. Seidner miteinander verbindet. Die beiden Pfarrer und Schriftsteller waren Zeitzeugen des kommunistischen Regimes, daher stellt diese Erzählung eine Brücke zur Vergangenheit dar.

Es ist leider so, dass nach bestimmten historischen Ereignissen viel Zeit vergeht, bis vielleicht jemand mit der Aufarbeitung beginnt ind dann sind oftmals die meisten Zeitzeugen nicht mehr am Leben. Aus diesem Grund sollte die junge Generation die Vergangenheit nicht nur aus Fachbüchern kennenlernen, sondern auch durch Befragung der Zeitzeugen. Eile ist geboten, denn es handelt sich um Vertreter der älteren Generation. Walther Gottfried Seidner, geboren 1938, verstarb im Sommer des Jahres 2018, Eginald Schlattner ist Jahrgang 1933 und nun schon über 85 Jahre alt.

Vor fast 20 Jahren erzählte Pfarrer Seidner Folgendes: Jedes Mal, wenn Bekannte oder Verwandte aus dem westlichen Ausland im kommunistischen Rumänien ankamen, fragten sie im Flüsterton, wo denn nun die Securitate sei. - „Überall“, antwortete der spitzzüngige evangelische Pfarrer. Das könnte die Wahrheit gewesen sein.

Es ist für uns jüngere Menschen beschwerlich mit dem heiklen Thema des rumänischen Sicherheitsdienstes umzugehen, schwierig ist es, zurechtzukommen mit den Verhaltensweisen 
der Generation vor uns im Sinne von „Verstrickung“ und „Ja nicht Ja“. Es ist zugleich Herausforderung und Verantwortung. Da jetzt viele der Zeitzeugen noch am Leben sind, kann jede Annäherung durch die Nachgeborenen von den direkt Betroffenen als beherzte Auseinandersetzung mit der kommunistischen Vergangenheit angesehen werden.

Der rumänische Sicherheitsdienst hat vierzig Jahre lang das Land und das rumänische Volk nicht nur allgegenwärtig im Auge gehabt, sondern noch brutaler im Griff; sein atheistisches Augenmerk war insondere auf die Kirchen und ihre Vertreter gerichtet. Die Menschen lechzten nach Freiheit; erst Ende 1989 kam der von allen erhoffte Umbruch durch die blutige Revolution im Dezember. Die Hinrichtung des Diktators und seiner allgewaltigen Frau und der zeitgleiche Fall des Eisernen Vorhangs veränderten Rumänien schlagartig. Man war plötzlich frei. Man konnte reden, eigene Meinungen äußern und vor allem gegen das politische Regime Aussagen machen. Doch bis heute gelingt es kaum, mit dieser Freiheit rechtens umzugehen.

Der Pfarrer und Schriftsteller Eginald Schlattner versucht in dem literarischen Bericht Ja nicht ja eines dieser brennenden Themen der jüngsten Vergangenheit zu vergegenwärtigen: Das Verhältnis zwischen Pfarrer und Securitate ${ }^{2}$, wobei er sich in dem besprochenen Text auf die evangelischen Pfarrer des Kirchenbezirks Hermannstadt bezieht.

Er tritt mit unwiderlegbaren Beweisen dagegen an, dass das, was über Jahrzehnte immer wieder gesagt worden ist, nicht stimmt: Alle sächsischen Pfarrer seien mit der Securitate verbunden gewesen.

Bei der ersten freien Pfarrerversammlung im Januar 1990 stellt sich für die fünfundfünfzig Pfarrer des Hermannstädter Kapitels heraus, dass einer, ein einziger Pfarrer, als Informant für den Geheimdienst gearbeitet hatte. Doch bedroht von der

2 „,securitate“ (rum. Sicherheit) war die volkstümlich abgekürzte rumänische Bezeichnung für den kommunistischen Geheimdienst. 
Securitate wurden alle und jeder. Wobei der Stolzenburger Geistliche Walther Seidner die Drohung des Geheimdienstes auf sich nimmt, bei seinem Nein zu riskieren, dass seine beiden Mädchen beim Schulweg auf der Straße totgefahren werden könnten.

Pfarrer haben von amtswegen Kontakt mit unzähligen Gemeindegliedern, also Zugang zu persönlichen Informationen über Familien aus ihrem unmittelbaren Umfeld. Weil die Macht der Securitate durch Informationen gesichert wurde, brauchte sie die Gottesmänner als geheime Informanten. Leicht gesagt, schwer zu handhaben, weil die meisten Pfarrer sich, wie gesagt, geweigert haben, sich in die Fallnetze des gegenseitigen Ausspionierens verstricken zu lassen.

Die Revolution 1989 dient als Einstieg für den Gesamttext, der sich auf konkrete Personen der siebenbürgisch-sächsischen Pfarrerwelt bezieht. Im Januar 1990 findet zum ersten Mal das monatliche Treffen der evangelischen Pfarrer des Hermannstädter Dekanats ohne das Auge der Securitate statt. Es ist sofort festzustellen, dass nach der blutigen Wende die bedrückende Wirklichkeit eine radikale Veränderung erfahren hat: „Bei dieser amtlichen Zusammenkunft schien alles wie sonst. Und nichts war wie einst." ${ }^{\text {3 }}$ Die Begegnung, die gewöhnlich unter direkter Beobachtung des Beauftragten der Kultusbehörde gestanden hatte, nimmt jetzt ihren ungewöhnlichen Lauf. Sie beginnt wie immer zur Überraschung aller Anwesenden mit einer Kaffeepause. Darauf folgt die eigentliche Sitzung.

Die erste Frage, die vom damaligen Dechanten ${ }^{4}$ Schuller gestellt wird, bezieht sich auf die persönliche Verbindung, die jeder Pfarrer zu dem rumänischen Sicherheitsdienst gehabt haben soll: ,Jetzt steht jeder auf, der hauptamtlich für die Securitate gearbeitet hat? ${ }^{* 5}$ Übrigens: Der Dechant wusste Bescheid.

3 Schlattner 2018, S. 37.

4 Auch Dekan oder Superintendent, in der evangelischen Kirche Vorsteher eines kirchlichen Verwaltungsbezirks.

5 Schlattner 2018, S. 41. 
Diese Frage schlägt wie eine Bombe ein und kann aus zwei Perspektiven gedeutet werden: Erstens wird allen Anwesenden klar, dass sie in einem neuen politischen System mit gesicherter Meinungsfreiheit leben, was sehr gewöhnungsbedürftig ist. Zweitens muss jeder sein Gewissen durchforsten und Rede und Antwort stehen, falls es eine Zusammenarbeit gegeben hat.

Die meisten der Beteiligten sind vor dem Bösen bewahrt geblieben, denn sie sind - trotz oft erschreckender Bedrängnisse - standhaft geblieben.

Das verpflichtende Band der politischen Abhängigkeit ist gerissen, die Pfarrer sind nach jahrelanger Beschattung und Bedrängung endlich frei. Es gibt kein schwarzes Auto mehr vor dem Haus, die Männerstimme am Telefon ist nicht mehr zu fürchten und vor allem können die alleswissenden ,besten Freunde' einem nichts mehr anhaben. Es ist vorbei; vorbei mit der Diktatur, vorbei mit derZeit der Angst und der Kompromisse. Entweder beichtet man die Wahrheit oder die Wahrheit kommt selber ans Licht. Es ist eine Frage der Zeit, denn alles geschieht zur rechten Zeit. Doch für einige ist es zu spät!

Die Antwort auf die Frage des Dechanten kommt von einem jungen Pfarrer, der die Zusammenarbeit mit der Securitate zugibt, aber auch einen weiteren interessanten Hinweis liefert: Er habe diesen Auftrag von seinem Vater übernommen, der nach Deutschland ausgereist sei. Diese Information scheint für das Verständnis seiner Position von Wichtigkeit zu sein: Es ist eine Art Verbindung zwischen den Generationen, die treu und langfristig, aber unfreiwillig mit dem Sicherheitsdienst kooperiert haben. Es handelt sich vielleicht, um es vorsichtig auszudrücken, um eine gewisse historische Tradition, die im Sinne des Überlebens auf ganz privater Ebene weitergeführt worden ist. Der junge Geistliche erklärt im Nachhinein, dass er durch die Ausführung dieses Auftrags seine Kollegen geschont habe: „Sonst hätte es einer von euch sein müssen!“ Trotz seines Alters und der Unerfahrenheit habe er versucht, seine Amtsbrüder 
nicht preiszugeben. Es ist tatsächlich möglich, dass er allein als Informant gedient hat, so dass die anderen Pfarrer ihre „Freiheit" behalten konnten. Was wohl in seinem Herzen gewesen ist, kann kein Mensch wissen. Es war für ihn bestimmt nicht leicht gewesen, ein Doppelleben zu führen. Man denke an die Gefahren, denen er ausgeliefert war: Ein Menschenleben hatte im damaligen Regime keinen Wert, wichtig war dieser Mensch nur als Informationsquelle, die zu funktionieren hatte.

Eginald Schlattner, der selber im Dezember 1957 verhaftet worden und fast zwei Jahre lang eingesperrt war, um dann unter Verlust der bürgerliche Rechte entlassen zu werden, der auch nachher schikaniert und bedroht worden war, erinnnert an die Worte „Voltaires“, wie Walther Seidner von Freunden genannt wurde, dass der Mensch nur das sehe, was vor seinen $\mathrm{Au}-$ gen stehe, dafür aber sehe Gott tief ins Herzen eines Menschen. $\mathrm{Ob}$ das genug ist, um den jungen Pfarrer zu entlasten? Schwer zu sagen, denn viele der ehemaligen Mitarbeiter der Securitate haben auch nach Jahrzehnten mit den Folgen ihrer freiwilligen bzw. unfreiwilligen Aussagen zu kämpfen. Der Schatten des Sicherheitsdienstes verfolgt sie ein Leben lang.

Die zweite Hauptfrage der Pastoralkonferenz bezieht sich auf die Betroffenen selbst, die durch die Securitate zu leiden hatten. Wenn die erste Frage Entsetzen und Stille ausgelöst hat, eröffnet der neue Sachverhalt eine andere Dimension. Plötzlich will jeder zu Wort kommen und seine persönliche Erfahrung mit den Leuten des Geheimdienstes preisgeben. Obwohl die Bedrängnis, ja Bedrohung groß gewesen war, verstanden alle Pfarrer, dass sie standhaft „nein“ sagen mussten, wenn es um die Bespitzelung des Nächsten ging. Im Sinne der biblischen Nächstenliebe galt es ,ja nicht ja“ zu sagen. Der evangelische Bischof Albert Klein unterstützte die Pfarrer in ihrem täglichen Kampf mit der Securitate, denn er wusste, was sich in jeder Gemeinde abspielte. Er nahm es sehr genau mit der Verwandtschaft in Jesus Christus und hatte einen persönlichen Bezug zu seinen Pfarrern. 
Der Schluss der Darstellung ist von besonderer Bedeutung für die Persönlichkeit, der dieser Text gewidmet ist. Walther Gottfried Seidner wurde - wie alle anderen - von dem Sicherheitsdienst bedroht, erpresst, seine Familie schwebte in Lebensgefahr. Andererseits: Im Falle einer Zusage hätte er bestimmt vielen Leute schaden müssen und wäre selber für immer angreifbar und erpressbar geworden. Mutig auf Gott setzend und die eventuell schlimmen Folgen für die Familie missachtend, hat er sich geweigert, Ja zu sagen. Er rettete dadurch andere Familien - und sein Gewissen. „Die gottlosen Männer“, die den Stolzenburger Pfarrer immer aufsuchten, hatten letztendlich die Gelegenheit, durch ihn Gottes Wort kennenzulernen.

Eginald Schlattners Text endet mit diesen Sätzen: „Pfarrer Seidner entließ die gottlosen Männer mit einem Bibelspruch, ihnen zugedacht wie jedem anderen Menschenkind unter Gottes Sonne: ,Wir wissen aber, dass denen, die Gott lieben, alle Dinge zum Besten dienen'.

Ja, er erlaubte sich eine winzige Wortverschiebung, wechselte ein Wort aus, nein: er tauschte zwei Buchstaben in demselben Wort um - und die Zusage Gottes bekam ein sehr anderes Gesicht: ,Wir wissen aber, dass denen, die Gott liebt, alle Dinge zum Besten dienen!" “6

Im Anschluss an die obigen Ausführungen soll nun Folgendes hinzugefügt werden: Die Festschrift für Walther Gottfried Seidner bedeutet nicht nur eine Huldigung an den Geehrten; sie enthält wertvolle, zugängliche Zeitzeugnisse, die zum Teil sehr persönlich formuliert sind. Da die Texte nicht dem Zwang der Wissenschaftlichkeit unterliegen, werden die Leser durch das Buch auf private Aspekte aus Seidners Leben aufmerksam gemacht. Die „Sammlung ergänzt das Bild dieses vielseitigen Künstlers" heißt es in Dietrich Galters Vorwort zum Band; das Verborgene kommt ans Licht und zeigt, dass Walther Gottfried Seidner vor allem der Menschlichkeit verpflichtet ist.

${ }^{6}$ Schlattner: Ja nicht ja. 2018, S. 51. 


\section{Literatur:}

Schlattner, Eginald: Ja nicht ja. Walther Gottfried Seidner zum 80. In: Andreea Dumitru; Sunhild Galter (Hgg.): Der siebenbürgische Voltaire. Walther Gottfried Seidner zum 80 Geburtstag. Sibiu/Hermannstadt: Honterus Verlag 2018, S. 36-51. 